Original Article

\title{
Prediction of falling risk after discharge in ambulatory stroke or history of fracture patients using Balance Evaluation Systems Test (BESTest)
}

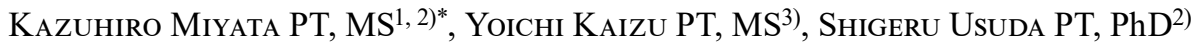 \\ 1) Department of Physical Therapy, Ibaraki Prefectural University of Health Science: 4669-2 \\ Ami-Machi, Inashiki-gun, Ibaraki 300-0394, Japan \\ 2) Graduate School of Health Sciences, Gunma University, Japan \\ 3) Department of Rehabilitation Center, Hidaka Hospital, Japan
}

\begin{abstract}
Purpose] The purpose of this study was to clarify the relationships between falls and sections of the Balance Evaluation Systems Test (BESTest) in patients with stroke or those with a history of fracture. [Subjects and Methods] This longitudinal study included 51 self-ambulatory inpatients. Balance was assessed 1 week prior to discharge using the BESTest, and the incidence of falls within 6 months after discharge was investigated. Relationships between falling and balance components were analyzed using the t-test or the Mann-Whitney $U$ test and receiver operating characteristic (ROC) curve analysis. [Results] Five subjects were dropped out before follow-up at 6 months. Falls were reported by 10 of the remaining 46 participants. Scores for two sections (Anticipatory Postural Adjustments and Sensory Orientation) were significantly lower in fallers than in non-fallers with stroke. Four of the six sections (Biomechanical Constraints, Anticipatory Postural Adjustments, Sensory Orientation, and Stability in Gait) showed areas under the ROC curves $>0.8(0.82,0.83,0.84$, and 0.81 , respectively). In patients with a history of fractures, all sections were not significantly different between fallers and non-fallers. [Conclusion] Anticipatory Postural Adjustments and Sensory Orientation sections of the BESTest were related to future occurrence of fall after discharge in self-ambulatory stroke patients.

Key words: Balance, Fall, Ambulatory stroke patients
\end{abstract}

(This article was submitted Dec. 2, 2017, and was accepted Jan. 9, 2018)

\section{INTRODUCTION}

Falls are the most serious and common medical problems suffered by stroke patients, elderly individuals, and persons with a history of fracture. Approximately $40 \%$ of people fall within the first year of a stroke ${ }^{1)}$, and one in three adults over the age of 65 years falls each year ${ }^{2}$. Balance is a critical skill for fall avoidance, and balance impairment is common among both stroke patients and older adults ${ }^{3)}$. Balance exercises can reduce falls ${ }^{4}$. A comprehensive balance assessment is recommended to identify impairments in postural control and optimize the design of balance exercise programs for fall prevention ${ }^{5)}$.

Balance control involves many physiological systems. Identifying the disordered systems underlying poor balance control is critical to establishing better intervention strategies for impaired balance. For this purpose, a comprehensive clinical balance test, the Balance Evaluation Systems Test (BESTest), was developed from a theoretical understanding of balance control systems ${ }^{6}$. The BESTest includes 36 items evaluating performance across six balance systems: Biomechanical Constraints (I), Stability Limits and Verticality (II), Anticipatory Postural Adjustments (III), Postural Responses (IV), Sensory Orientation (V), and Stability in Gait (VI). Since its introduction in 2009, the BESTest has been increasingly used for evaluating balance function in various populations. The BESTest offers good reliability and validity ${ }^{6-9}$, and it has been used to evaluate

*Correspondence author. Kazuhiro Miyata (E-mail: ipukazuhiro@yahoo.co.jp)

(C2018 The Society of Physical Therapy Science. Published by IPEC Inc.

(c) (1) $\odot$ This is an open-access article distributed under the terms of the Creative Commons Attribution Non-Commercial No Derivacc. 
balance deficits under various pathologic conditions, including stroke ${ }^{7}$, Parkinson's disease (PD) ${ }^{8)}$, and type 2 diabetes mellitus ${ }^{9)}$. Moreover, the BESTest has high responsiveness, with no floor or ceiling effects in patients with subacute stroke ${ }^{10)}$. In addition, the BESTest has been identified as the only standardized balance measure to evaluate all components of balance consistent with established conceptual models of the "Systems Framework of Postural Control"11).

Several studies have demonstrated that the BESTest is useful as a fall prediction balance instrument ${ }^{8,9,12-14)}$. The BESTest has moderate fall prediction accuracy in people with $\mathrm{PD}^{8,12)}$, type 2 diabetes mellitus ${ }^{9)}$, and older participants ${ }^{13}$, 14). We have recently reported that the BESTest offered the same predictive accuracy as the Berg Balance Scale (BBS), which has been conventionally used for patients who have been discharged from hospital convalescent rehabilitation wards ${ }^{15}$. While the previous reports have provided useful information on total scores from the BESTest, relationships between sections and falls have been little studied. Different sections of the BESTest have been reported to show significant decreases in patients with knee osteoarthritis ${ }^{16)}$ and chronic obstructive pulmonary disease ${ }^{17)}$ compared to age-matched healthy subjects.

In this study, we focused on patients with stroke and those with a history of fracture who are diseases occupying more than $80 \%$ of the convalescent rehabilitation ward. Both disease ambulatory people who can walk also have a risk of falling. Therefore, it is important to understand detailed problem of balance disorder before discharge. Using sections of the BESTest may thus help clarify the structure of balance disorders and improve rehabilitation interventions.

The purpose of this study was to clarify the relationships between falls and sections of the BESTest in patients with stroke and those with a history of fracture who were discharged from a hospital convalescent rehabilitation ward. We hypothesized that different sections of the BESTest have the relationship with falls in both stroke and those with a history of fracture patients. There should be sections that are strongly related to falls and have high prediction accuracy.

\section{SUBJECTS AND METHODS}

The research protocol was approved by the Ethics Committee of Hidaka Hospital and Hidaka Rehabilitation Hospital (No. $53,110902)$. All patients provided written informed consent prior to participating. This study was conducted in accordance with the Declaration of Helsinki.

Sample size calculations for the study were based on a power of 0.80 and an alpha level of 0.05 . The receiver operating characteristic (ROC) curve based on a $30 \%$ faller rate, and the area under the curve (AUC) $=0.85$ required 19 participants for each disease.

The study recruited 51 consecutive patients admitted to and then discharged from two hospital convalescent rehabilitation wards between May 2011 and October 2013. The inclusion criteria were independence in ambulation (functional ambulation category $[\mathrm{FAC}] \geq 4$ ) and mild balance disorder (BBS $>45)$. Exclusion criteria were as follows: blindness or severe visual impairments affecting the ability to walk independently; inability to follow instructions; cognitive impairments (Hasegawa Dementia Scale-Revised [HDS-R] $>20$ ); or reduced activities of daily living due to pain. The underlying disease was hemiparesis in 20 patients and fracture in 31 patients.

This was a longitudinal study that investigated the occurrence of falls over 6 months after discharge. Baseline assessments were performed within 1 week prior to discharge from the hospital. Each subject performed the BESTest. The occurrence of falls within 6 months after discharge from the hospital was determined by follow-up telephone calls. A fall was defined as "unintentionally coming to the ground or some lower level for some reason other than as a consequence of sustaining a violent blow, loss of consciousness, or sudden onset of paralysis as in stroke or epileptic seizure"18). A faller was defined as someone who reported 1 or more falls in the prior 6 months.

The BESTest contains 27 items, with some items consisting of two or four subitems (e.g., separate items for left and right sides), for a total of 36 items. Each item is rated using a 4-level rating scale ranging from 0 (severe balance impairment) to 3 (no balance impairment). The score is measured as a percentage of the maximum possible score of 108 points. Scores are converted to percentages, with higher scores indicating better balance performance ${ }^{6)}$. The reliability of the BESTest has been confirmed in stroke patients ${ }^{7)}$ and community-dwelling older adults ${ }^{13)}$.

Statistical analyses were performed separately for stroke and fracture patients. The normality of data for sections of the BESTest was examined using the Shapiro-Wilk test. Fallers and non-fallers with each disease were compared using the independent sample t-test for normally distributed sections and the Mann-Whitney $U$ test for non-normally distributed sections. When significant differences on the performance of sections were found between fallers and non-fallers, the effect size (ES) was calculated. The ES was Cohen's d for the independent sample t-test and $\mathrm{r}$ for the Mann-Whitney U test; the ES was considered small $(\mathrm{d}=0.2, \mathrm{r}=0.1)$, medium $(\mathrm{d}=0.5, \mathrm{r}=0.3)$, or large $\left.(\mathrm{d}=0.8, \mathrm{r}=0.5)^{19}\right)$.

The AUC of the ROC curve was used to determine the relative performance of the sections of the BESTest for classifying participants as fallers and non-fallers. An AUC value $>0.9$ was interpreted as high accuracy, $0.7-0.9$ as moderate accuracy, $0.5-0.7$ as low accuracy, and $<0.5$ as due to chance ${ }^{20)}$. Sensitivity and specificity values were calculated. The cutoff was determined as that value providing the best balance between high sensitivity and high specificity. A positive likelihood ratio $(\mathrm{LR}+)$ and a negative likelihood ratio (LR-) were also calculated for each section of the BESTest. An LR+ greater than 5 and an LR- less than 0.2 indicate that the section of the BESTest is useful due to its high probability of correctly identifying participants with and without falls.

The statistical software used was IBM SPSS Statistics (Version 24.0; IBM Corp, Armonk, NY, USA). Unless otherwise 
indicated, the level of significance was set at $\mathrm{p}<0.05$.

\section{RESULTS}

Of the 51 participants enrolled at baseline, five dropped out because they moved and could not be contacted, or because they developed an unrelated illness. One of these five was a stroke patient, and the others were fracture patients. Data from the remaining 46 participants were analyzed. Ten participants reported falling, yielding a fall rate of $21.7 \%$. Participant characteristics, gait ability, and balance scores are presented in Table 1.

Table 2 presents the scores for each section of the BESTest in fallers and non-fallers with stroke. The scores for three sections, Biomechanical Constraints (I), Postural Response (IV), and Stability in Gait (VI), were normally distributed. The scores for two sections of the BESTest were significantly lower in fallers than in non-fallers. For the Anticipatory Postural Adjustments (III) and Sensory Orientation (V) sections, the ES was large, with $\mathrm{r}=0.50$ and $\mathrm{r}=0.51$, respectively. Table 3 presents the scores for each section in fallers and non-fallers with fractures. Scores for three sections, Anticipatory Postural Adjustments (III), Postural Response (IV), and Stability in Gait (VI), were normally distributed. None of the sections of the BESTest were significantly different between fallers and non-fallers.

ROC analyses were performed only for stroke patients where there was a significant difference between fallers and nonfallers. Results of the AUC of ROC curve analyses for each section of the BESTest are presented in Fig. 1 and Table 4.

Table 1. Characteristics and clinical measures of the participants

\begin{tabular}{lcccccc}
\hline & \multicolumn{3}{c}{ Stroke } & \multicolumn{3}{c}{ Fracture } \\
\cline { 2 - 7 } & $\begin{array}{c}\text { Over all } \\
(\mathrm{n}=19)\end{array}$ & $\begin{array}{c}\text { Non-fallers } \\
(\mathrm{n}=14)\end{array}$ & $\begin{array}{c}\text { Fallers } \\
(\mathrm{n}=5)\end{array}$ & $\begin{array}{c}\text { Over all } \\
(\mathrm{n}=27)\end{array}$ & $\begin{array}{c}\text { Non-fallers } \\
(\mathrm{n}=22)\end{array}$ & $\begin{array}{c}\text { Fallers } \\
(\mathrm{n}=5)\end{array}$ \\
\hline Age (years) & $63.0 \pm 13.3$ & $62.9 \pm 14.3$ & $63.0 \pm 9.9$ & $76.0 \pm 8.4$ & $75.8 \pm 9.3$ & $78.2 \pm 5.9$ \\
Gender (female/male) & $8 / 11$ & $6 / 8$ & $2 / 3$ & $20 / 7$ & $18 / 4$ & $2 / 3$ \\
Time since onset (days) & $87.9 \pm 38.6$ & $82.4 \pm 31.9$ & $111.0 \pm 43.3$ & $76.4 \pm 29.3$ & $81.3 \pm 30.5$ & $74.6 \pm 21.7$ \\
$\begin{array}{l}\text { Disease type (ischemic/hemorrhagic } \\
\text { or femoral/vertebral) }\end{array}$ & $11 / 8$ & $7 / 7$ & $4 / 1$ & $17 / 10$ & $14 / 8$ & $3 / 2$ \\
FAC (scores, 4/5) & $14 / 5$ & $10 / 5$ & $4 / 0$ & $22 / 5$ & $17 / 5$ & $5 / 0$ \\
BESTest (range, 0-100\%) & $77.4 \pm 13.6$ & $81.4 \pm 7.9$ & $65.2 \pm 18.1$ & $78.9 \pm 9.2$ & $79.2 \pm 8.8$ & $72.8 \pm 9.8$ \\
\hline
\end{tabular}

Values are given as means \pm SD, n, or as otherwise indicated. FAC: functional ambulation category; BESTest: Balance Evaluation Systems Test.

Table 2. Score on each section of the BESTest in fallers and non-fallers with stroke

\begin{tabular}{lllc}
\hline & \multicolumn{1}{c}{ Non-fallers $(\mathrm{n}=14)$} & \multicolumn{1}{c}{ Fallers $(\mathrm{n}=5)$} & $\mathrm{p}$ \\
\hline I. Biomechanical Constraints & $85.7 \pm 9.4(81.0-91.1)$ & $64.0 \pm 22.9(35.6-92.4)$ & 0.101 \\
II. Stability Limits and Verticality & $84.6 \pm 6.1(81.0-88.1)$ & $79.0 \pm 18.3(56.3-101.8)$ & 0.500 \\
III. Anticipatory Postural Adjustments & $75.8 \pm 13.0(68.3-83.3)$ & $53.3 \pm 21.0(27.2-79.4)$ & 0.034 \\
IV. Postural Responses & $76.2 \pm 17.6(66.0-86.4)$ & $68.9 \pm 29.8(31.9-105.9)$ & 0.517 \\
V. Sensory Orientation & $88.6 \pm 9.9(82.8-94.3)$ & $74.7 \pm 8.7(63.9-85.5)$ & 0.026 \\
VI. Stability in Gait & $80.3 \pm 11.0(74.0-86.6)$ & $52.4 \pm 32.1(12.5-92.3)$ & 0.125 \\
\hline
\end{tabular}

Values are given as means \pm SD (95\% confidence interval). BESTest: Balance Evaluation Systems Test.

Table 3. Score on each section of the BESTest in fallers and non-fallers with fractures

\begin{tabular}{lllc}
\hline & \multicolumn{1}{c}{ Non-fallers $(\mathrm{n}=22)$} & \multicolumn{1}{c}{ Fallers $(\mathrm{n}=5)$} & $\mathrm{p}$ \\
\hline I. Biomechanical Constraints & $80.9 \pm 12.6(75.3-86.5)$ & $74.7 \pm 14.4(56.8-92.6)$ & 0.208 \\
II. Stability Limits and Verticality & $85.1 \pm 5.9(82.4-87.7)$ & $85.7 \pm 3.4(81.5-89.9)$ & 0.786 \\
III. Anticipatory Postural Adjustments & $74.4 \pm 16.7(67.0-81.8)$ & $65.6 \pm 17.3(44.1-87.0)$ & 0.338 \\
IV. Postural Responses & $72.2 \pm 13.5(66.2-78.2)$ & $57.8 \pm 28.5(22.4-93.2)$ & 0.095 \\
V. Sensory Orientation & $91.2 \pm 8.6(87.4-95.0)$ & $96.0 \pm 6.0(88.6-103.4)$ & 0.314 \\
VI. Stability in Gait & $74.0 \pm 16.5(66.7-81.4)$ & $61.0 \pm 24.6(30.4-91.6)$ & 0.275 \\
\hline
\end{tabular}

Values are given as means \pm SD (95\% confidence interval). BESTest: Balance Evaluation Systems Test. 
The AUC was moderate (0.7-0.9) for the Biomechanical Constraints (I), Anticipatory Postural Adjustments (III), Sensory Orientation (V), and Stability in Gait (VI) sections. For sensitivity and specificity, Anticipatory Postural Adjustments (III) and Stability in Gait (VI) sections had values larger than 0.7. In addition, the LR+ and LR- analyses indicated that the Stability in Gait (VI) section was the most appropriate section for classifying participants with and without falls.

\section{DISCUSSION}

To the best of our knowledge, this is the first study to investigate the relationships between falls and sections of the BESTest in people with strokes and those with fractures. Four sections of the BESTest showed acceptable ability to differentiate between fallers and non-fallers in stroke patients, with similar AUCs. In particular, the Anticipatory Postural Adjustments (III) and Sensory Orientation (V) sections were important, because there was also a significant difference between fallers and non-fallers. These results generally supported our hypothesis to stroke patients, suggesting the importance of conducting a detailed assessment of balance disorder by using BESTest for the even ambulatory stroke patient to prevent falls.

Asymmetry and weight-shift on standing ${ }^{21)}$, disturbance load response and anticipatory postural adjustment ${ }^{22}$, and postural control of dual tasks ${ }^{23}$ are characteristics of postural control of stroke patients. The results of the present study suggest the importance of two balance components, anticipatory postural adjustment and maintaining standing posture depending on sensory function. First, the Anticipatory Postural Adjustments (III) section of the BESTest includes tasks that require active movement of the body's center of mass in anticipation of a postural transition from one body position to another, such as rise to toes, stand on one leg, and alternate stair touching ${ }^{6}$ ). The Anticipatory Postural Adjustments (III) section seems to have greater difficulty, because fallers and non-fallers had lower average scores than on the other sections. Moreover, the fall prediction cutoff point was the lowest. Previous studies have reported response delay and decreased muscle activity of the hemiparesis side trunk and lower limb using electromyography analysis ${ }^{22}{ }^{24}$. Although it is difficult for stroke patients to acquire abilities, preventing falls is important. Second, the Sensory Orientation (V) section of the BESTest identifies any

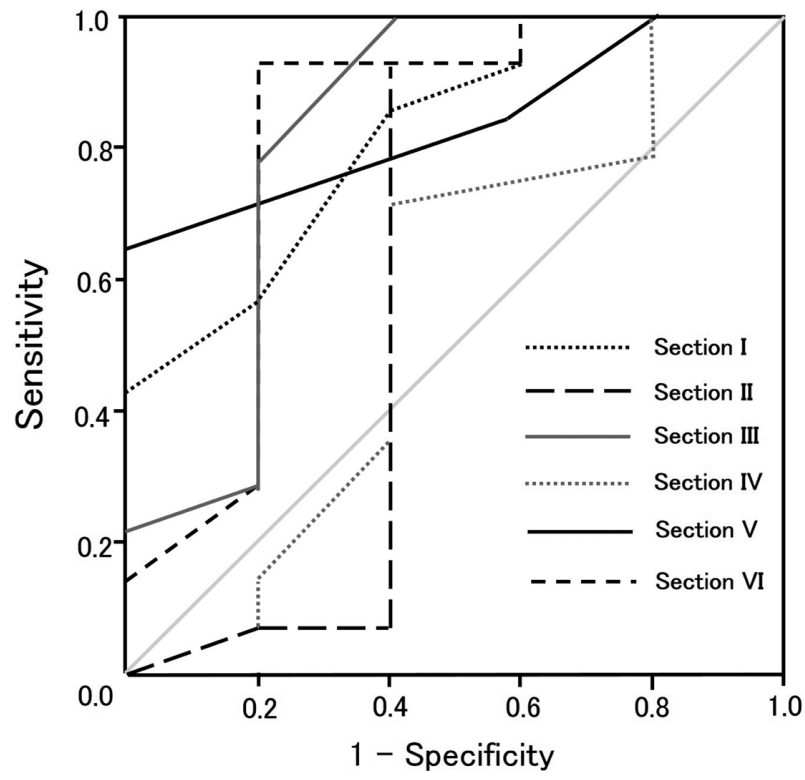

Fig. 1. Receiver operating characteristic (ROC) curve for the section of the BESTest.

Table 4. Predictive values of the sections of the BESTest in patients with stroke

\begin{tabular}{lccccrr}
\hline & AUC $(95 \% \mathrm{CI})$ & Cutoff point $(\%)$ & Sensitivity & Specificity & LR+ & LR- \\
\hline I. Biomechanical Constraints & $0.82(0.61-1.00)$ & 76.7 & 0.86 & 0.60 & 2.14 & 0.30 \\
II. Stability Limits and Verticality & $0.61(0.21-1.00)$ & 78.6 & 0.93 & 0.60 & 2.32 & 0.35 \\
III. Anticipatory Postural Adjustments & $0.83(0.56-1.00)$ & 63.9 & 0.79 & 0.80 & 3.92 & -0.02 \\
IV. Postural Responses & $0.56(0.22-0.90)$ & 69.5 & 0.71 & 0.60 & 1.79 & 0.16 \\
V. Sensory Orientation & $0.84(0.66-1.00)$ & 83.3 & 0.64 & 1.00 & Unable to calculate & -0.56 \\
VI. Stability in Gait & $0.81(0.54-1.00)$ & 69.1 & 0.93 & 0.80 & 4.64 & 0.13 \\
\hline
\end{tabular}

BESTest: Balance Evaluation Systems Test; AUC: area under the curve; CI: confidence interval; LR: likelihood ratio 
increase in body sway during stance associated with altering visual or surface somatosensory information for control of standing balance. The task consists of the modified Clinical Test of Sensory Integration for Balance (CTSIB) and involves standing on a slope with eyes closed ${ }^{6,25)}$. In stroke patients, standing postural control may be visually dependent due to somatosensory impairment, muscle weakness, and asymmetric muscle tone ${ }^{26)}$. In a study of shaking of the center of pressure, it appeared to depend on visual inputs, because the shaking increased with the eyes closed compared with opened eyes ${ }^{21)}$. It is interesting that the decline of this ability is more related to falls than other balance components.

The Biomechanical Constraints (I) and Stability in Gait (VI) sections of the BESTest had a moderate AUC, but there were no significant differences between the fallers and non-fallers. In a previous study of PD, the AUC of the total score of the BESTest was 0.84 to $0.89^{8,12}$, which was comparable to the four sections of the present study. Further, there was a significant difference in all sections of the BESTest in fallers and non-fallers with PD. This is different from the stroke patients of the present study. Even neurological disorders tend to differ depending on the disease state and period, and further investigation is necessary.

The Stability Limits and Verticality (II) and Postural Responses (IV) sections of the BESTest had little relevance to falls. The Stability Limits and Verticality (II) section may have been an easy task for the present patients with stroke; because this section included tasks in the sitting position, the mean value and cutoff point were also high. This section is not involved in falls, although it might represent basic components of balance construction for independence of walking. As for the Postural Responses (IV) section, the AUC was close to the chance level, and the $95 \%$ confidence interval of the fallers was wide. The stepping response is an important balance component, but in this study, there is a possibility that the influence of individual differences became large because there were few participants. Whether this is a characteristic of stroke needs further study.

As for patients with fractures, there were no significant differences in all sections of the BESTest between fallers and non-fallers. This result was different from our hypothesis. In previous studies of elderly people living in the community, balance was a factor related to falls, and the AUC of the BESTest total score was shown to have moderate prediction accuracy, from 0.71 to $0.74^{13,14)}$. Most of the participants were elderly people aged 65 years and older, who were hospitalized due to a fracture caused by a fall. The reason why the balance component related to the fall was not a factor may be that the participants were limited to those with mild balance disorders. In the previous study, 45 points on the BBS was regarded as the cutoff point for prediction of falls ${ }^{27}$ ), but in the present study, a higher score was identified. As for the characteristics of the sections of the BESTest, the fallers exceeded the score of the non-fallers in two sections, Stability Limits and Verticality (II) and Sensory Orientation (V). Because of the high scores, it is suggested that they are indispensable balance components for self-ambulatory persons. In the future, it will be necessary to investigate patients with a wide range of abilities, clarifying the causes of falls.

This study was somewhat limited by its small sample size. In addition, participants in the present study were independent walkers with mild balance disorders. In future studies, surveying the presence or absence of injuries and the number of falls, improving the accuracy with which falls are determined, increasing the number of participants, and extending the time period will be important.

In summary, the present study demonstrated that the Anticipatory Postural Adjustments (III) and Sensory Orientation (V) sections of the BESTest were related to falls in self-ambulatory stroke patients. Further studies are recommended to confirm the effects of the sections of the BESTest in the prevention of falls in stroke patients.

\section{Conflict of interest}

None.

\section{ACKNOWLEDGEMENT}

The authors would like to thank the participating facilities and rehabilitation staff for their cooperation and assistance in data collection.

\section{REFERENCES}

1) Langhorne P, Stott DJ, Robertson L, et al.: Medical complications after stroke: a multicenter study. Stroke, 2000, 31: 1223-1229. [Medline] [CrossRef]

2) Tinetti ME, Speechley M, Ginter SF: Risk factors for falls among elderly persons living in the community. N Engl J Med, 1988, 319: 1701-1707. [Medline] [CrossRef]

3) Tyson SF, Hanley M, Chillala J, et al.: Balance disability after stroke. Phys Ther, 2006, 86: 30-38. [Medline] [CrossRef]

4) Sherrington C, Whitney JC, Lord SR, et al.: Effective exercise for the prevention of falls: a systematic review and meta-analysis. J Am Geriatr Soc, 2008, 56: 2234-2243. [Medline] [CrossRef]

5) Horak FB: Postural orientation and equilibrium: what do we need to know about neural control of balance to prevent falls? Age Ageing, 2006 , 35: ii7-ii11. [Medline] [CrossRef]

6) Horak FB, Wrisley DM, Frank J: The Balance Evaluation Systems Test (BESTest) to differentiate balance deficits. Phys Ther, 2009, 89: 484-498. [Medline] 
[CrossRef]

7) Chinsongkram B, Chaikeeree N, Saengsirisuwan V, et al.: Reliability and validity of the Balance Evaluation Systems Test (BESTest) in people with subacute stroke. Phys Ther, 2014, 94: 1632-1643. [Medline] [CrossRef]

8) Leddy AL, Crowner BE, Earhart GM: Functional gait assessment and balance evaluation system test: reliability, validity, sensitivity, and specificity for identifying individuals with Parkinson disease who fall. Phys Ther, 2011, 91: 102-113. [Medline] [CrossRef]

9) Marques A, Silva A, Oliveira A, et al.: Validity and relative ability of 4 balance test to identify fall status of older adults with type 2 diabetes. J Geriatr Phys Ther, 2017, 40: 227-232. [Medline] [CrossRef]

10) Chinsongkram B, Chaikeeree N, Saengsirisuwan V, et al.: Responsiveness of the Balance Evaluation Systems Test (BESTest) in people with subacute stroke. Phys Ther, 2016, 96: 1638-1647. [Medline] [CrossRef]

11) Sibley KM, Beauchamp MK, Van Ooteghem K, et al.: Using the systems framework for postural control to analyze the components of balance evaluated in standardized balance measures: a scoping review. Arch Phys Med Rehabil, 2015, 96: 122-132.e29. [Medline] [CrossRef]

12) Duncan RP, Leddy AL, Cavanaugh JT, et al.: Comparative utility of the BESTest, mini-BESTest, and brief-BESTest for predicting falls in individuals with Parkinson disease: a cohort study. Phys Ther, 2013, 93: 542-550. [Medline] [CrossRef]

13) Marques A, Almeida S, Carvalho J, et al.: Reliability, validity, and ability to identify fall status of the balance evaluation systems test, mini-balance evaluation systems test, and brief-balance evaluation systems test in older people living in the community. Arch Phys Med Rehabil, 2016, 97: 2166-2173.e1. [Medline] [CrossRef]

14) Yingyongyudha A, Saengsirisuwan V, Panichaporn W, et al.: The Mini-Balance Evaluation Systems Test (Mini-BESTest) demonstrates higher accuracy in identifying older adult participants with history of falls than do the BESTest, berg balance scale, or timed up and go test. J Geriatr Phys Ther, 2016, 39: 64-70. [Medline] [CrossRef]

15) Miyata K, Koizumi M, Iwai Y, et al.: Score distribution and fall prediction using the Balance Evaluation Systems Test (BESTest), Mini-BESTest, and BriefBESTest: a prospective cohort study. Phys Ther Jpn, 2016, 43: 118-126 (in Japanese).

16) Tamura T, Otaka Y, Konno S, et al.: The impaired balance systems identified by the BESTest in order patients with knee osteoarthritis. PM R, 2016, 8: 869-875. [Medline] [CrossRef]

17) Beauchamp MK, Sibley KM, Lakhani B, et al.: Impairments in systems underlying control of balance in COPD. Chest, 2012, 141: 1496-1503. [Medline] [CrossRef]

18) Gibson MJ: Falls in later life. In: Improving the health of older people; a world view. New York: Oxford University Press, 1990.

19) Cohen J: Statistical power analysis for behavioral sciences, 2nd ed. Hillsdale: Lawrence Erlbaum Associates, 1982.

20) Akobeng AK: Understanding diagnostic tests 3: receiver operating characteristic curves. Acta Paediatr, 2007, 96: 644-647. [Medline] [CrossRef]

21) Marigold DS, Eng JJ: The relationship of asymmetric weight-bearing with postural sway and visual reliance in stroke. Gait Posture, 2006, 23: 249-255. [Medline] [CrossRef]

22) Holt RR, Simpson D, Jenner JR, et al.: Ground reaction force after a sideways push as a measure of balance in recovery from stroke. Clin Rehabil, 2000, 14: 88-95. [Medline] [CrossRef]

23) Hyndman D, Ashburn A, Yardley L, et al.: Interference between balance, gait and cognitive task performance among people with stroke living in the community. Disabil Rehabil, 2006, 28: 849-856. [Medline] [CrossRef]

24) Slijper H, Latash ML, Rao N, et al.: Task-specific modulation of anticipatory postural adjustments in individuals with hemiparesis. Clin Neurophysiol, 2002, 113: 642-655. [Medline] [CrossRef]

25) Shumway-Cook A, Horak FB: Assessing the influence of sensory interaction of balance. Suggestion from the field. Phys Ther, 1986, 66: 1548-1550. [Medline] [CrossRef]

26) Bonan IV, Colle FM, Guichard JP, et al.: Reliance on visual information after stroke. Part I: Balance on dynamic posturography. Arch Phys Med Rehabil, 2004, 85: 268-273. [Medline] [CrossRef]

27) Berg K: Measuring balance in the elderly: preliminary development of an instrument. Physiother Can, 1989, 41: 304-311. [CrossRef] 